\title{
To prepare or not to prepare? When preparation of a response in Task 2 induces extra performance costs in Task 1
}

\author{
Moritz Durst $^{1}$ (D) $\cdot$ Rolf Ulrich $^{1} \cdot$ Markus Janczyk $^{1,2}$ (D)
}

Published online: 18 March 2019

(C) The Psychonomic Society, Inc. 2019

\begin{abstract}
In dual tasking, the no-go backward crosstalk effect (BCE) means that processing of Task 1 takes longer when Task 2 does not require a response (no-go trial) than when it requires a response (go trial). Thus, contrary to the usual observation, giving two successive responses counterintuitively reduces instead of increases performance costs for Task 1 . Results from recent studies are in line with the notion that the no-go BCE reflects response inhibition, which is required to overcome an already prepared go response in Task 2, but which also spills over to motor execution in Task 1. No direct test of this hypothesis, however, has been carried out so far, and hence the present study was designed to fill this gap. The result of this study with $n=48$ participants revealed that a no-go Task 2 impeded Task 1 performance when preparation of the Task 2 response was encouraged, but facilitated Task 1 performance when preparation of the Task 2 response was not encouraged.
\end{abstract}

Keywords Dual task $\cdot$ Backward crosstalk $\cdot$ Go/no-go task $\cdot$ Response preparation

Performing movements in two motor tasks simultaneouslythat is, dual tasking — can be very difficult and often comes at the cost of performing one or both movements slower and/or producing more errors. Just imagine learning to play drums where you have to simultaneously coordinate contrary movements of both arms and feet. As most dual-tasking research and popular opinions nowadays focus on the difficulties of dual tasking, situations in which negative consequences are reduced when two instead of only one movement are carried out are often overlooked. However, this happens in no-go backward crosstalk experiments.

Miller (2006) was the first to combine a manual two-choice Task 1 with a go/no-go Task 2 (see Donders, 1969). For example, in his Experiment 1, participants were instructed to respond to the identity of a letter by pressing a key with their left index or middle finger in Task 1, and to respond to the pitch of a tone by pressing a key with their right index finger

Moritz Durst

moritz.durst@psycho.uni-tuebingen.de

1 Department of Psychology, Eberhard Karls University of Tübingen, Schleichstraße 4, 72076 Tübingen, Germany

2 Present address: Department of Psychology, University of Bremen, Bremen, Germany (go trials) or by withholding the response (no-go trials) in Task 2. Task 1 response times (RTs) were shorter when Task 2 required a response relative to when it did not. This no-go backward crosstalk effect (no-go BCE) is an example of how dual-task costs are actually reduced when two motor tasks have to be carried out (see also Ko \& Miller, 2014; Miller \& Durst, 2014, 2015), even though the addition of a second task certainly impedes performance of the first task overall (i.e., general dual-task costs are present).

The most often invoked explanation for the no-go BCE is that inhibition is required to withhold an already prepared Task 2 response in no-go trials, whereby some inhibition spills over to Task 1 and thus prolongs RT1s in no-go trials (Janczyk \& Huestegge, 2017; Miller, 2006). In addition, recent results from a mental chronometry study (Durst \& Janczyk, 2018) also suggest that indeed motor execution in Task 1 is prolonged in Task 2 no-go trials, thus lending additional support for the preparation hypothesis. As an alternative explanation, Röttger and Haider (2017) suggested that the no-go BCE is based on automatic response feature activation. For instance, such response features could be response effects that seem to play an important role in the selection of no-go responses as well (Kühn, Elsner, Prinz, \& Brass, 2009). In this sense, the sensory consequence of a go and no-go response would be incompatible, which could impair Task 1 performance in no-go trials. Alternatively, the incompatibility of 
abstract "go-representations" versus "no-go representations" could impair Task 1 performance. Importantly, according to this hypothesis, the mere perception of a no-go stimulus in Task 2 should automatically activate the (incompatible) nogo representation and thereby impair performance of Task 1 (which always involves a go representation). In sum, and in contrast to the abovementioned preparation hypothesis, the no-go BCE would then emerge entirely independent from the preparatory status of the Task 2 response.

Even though the larger part of evidence indirectly supports the preparation hypothesis, a direct test of the competing explanations still lacks. The present study fills this gap. To this end, a two-choice Task 1 was combined with a choice/no-go Task 2, which either required one of two go responses or to withhold the response (no-go response). The critical manipulation was that preparation of a Task 2 go response was either encouraged or not by varying the frequency of the Task 2 go stimuli, keeping everything else equivalent. More specifically, in one half of the blocks, both go stimuli occurred equally often (neutral blocks). Thus, participants would be less encouraged to prepare one or the other response in advance, and consequently no or even a reversed no-go BCE was expected, because there was nothing to inhibit in case of a no-go stimulus (see also Janczyk \& Huestegge, 2017, for this observation). In the other half of blocks, one go stimulus occurred in $90 \%$ of the go trials (biased blocks), thereby encouraging preparation of the more often required Task 2 response. In this case, the preparation hypothesis predicts that the no-go BCE should reemerge in these biased blocks. Although previous research has supported the view that the no-go BCE has its locus in the motor stage of Task 1 (Durst \& Janczyk, 2018), the role of preparation in Task 2 (and the required inhibition) has not been addressed directly so far. Yet evidence for the preparation hypothesis would not only help to better understand the no-go BCE as such, but also to further delineate it from compatibility-based BCEs (Hommel, 1998). These latter BCEs occur when both tasks overlap, for example, in their spatial response features, are caused by automatic response feature activation in Task 2, and have their locus during Task 1 response selection instead of motor execution (see Janczyk, Renas, \& Durst, 2018; for a direct comparison of both kinds of BCEs see, Durst \& Janczyk, 2019).

\section{Method}

\section{Participants}

Forty-eight people (28 female) from the Tübingen (Germany) area, aged 19 to 64 years $(M=23.21$ years, $S D=6.56)$, participated for monetary compensation $(8 €)$ or course credit. All participants provided written informed consent before the experiment and reported normal or corrected-to-normal vision.
According to Röttger and Haider (2017, p. 602) their Experiment 1 and Miller's (2006) Experiment 1 exhibited large effect sizes $\left(\boldsymbol{\eta}_{\mathbf{p}}^{2}>.5\right)$. The effect of interest in our experiments is a $2 \times 2$ interaction of two repeated-measures which can be broken down to a (paired) $t$ test, and we (conservatively) assume a medium effect size of $d_{z}=0.5$ (Cohen, 1988). Power analysis using the function power.t.test() of $\mathrm{R}$ software yielded a required sample size of $n \approx 44$ participants to achieve $1-\beta=.90$, with $\alpha=.05$.

\section{Apparatus and stimuli}

A standard PC was used for stimulus presentation and response collection. Stimuli and instructions were presented on a 17-inch CRT monitor. Stimuli were the letters $X$ and $O$ colored in red, green, and blue and presented in the center of an otherwise black screen. The identity of the letter served as Stimulus 1 (S1), the color of the letter served as Stimulus 2 (S2). Responses to S1 were given via a manual key press of the left index or middle finger (R1). Responses to S2 were given via a manual key press of the right index or middle finger, or by withholding the response (R2). Custom-made keys were used to collect all responses, and two keys each were placed to the left and to the right of the participant.

\section{Task and procedure}

The trial structure is illustrated in Fig. 1. Task 1 was to respond to S1 with a manual key press of the left index or middle finger, and Task 2 was either to respond to S2 (go trial) with an index or middle finger key press of the right hand or to withhold the response (no-go trial).

Each trial started with a white fixation cross $(250 \mathrm{~ms})$, followed by a blank screen $(250 \mathrm{~ms})$. Subsequently, the colored letter was presented at the center of the screen for a maximum of $4,000 \mathrm{~ms}$ or until R1 and R2 were registered. The next trial started after an intertrial interval (ITI) of 1,000 ms. In case of wrong responses or general errors (no response within 4,000 ms following stimulus onset, responses in the wrong order, response with a wrong key, and so on), specific error feedback was provided for 1,000 ms before the ITI.

Participants first performed a short familiarization block of 20 randomly drawn trials, which was followed by eight experimental blocks of 60 trials each. All trials were presented in a random order. Throughout the experiment, the S2 associated with the no-go response occurred in one third of the trials (nogo trials), while an S2 associated with a go response of the right hand occurred in the remaining two thirds of all trials (go trials). In neutral blocks, both S2 associated with go responses occurred equally often (i.e., 20 times), whereas in the biased blocks one S2 associated with either the right index or middle finger response occurred in $90 \%$ of all go trials (i.e., 36 times). 


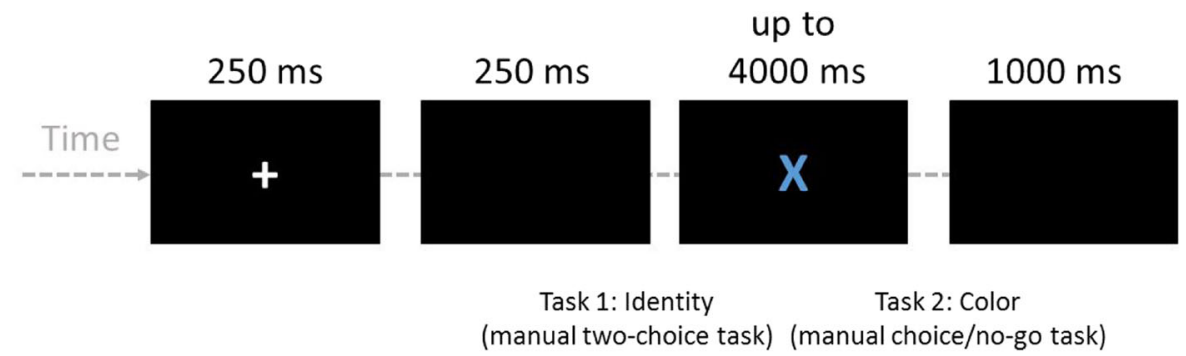

\section{Go-trial}

\section{No-go-trial}

Fig. 1. Trial structure for go and no-go trials. Participants first responded to the identity of the letter in a manual two-choice task with the left hand and subsequently to the color of the letter in a manual choice/no-go task with the right hand. In this particular example, an $X$ calls for a response

Participants received written instructions that informed them about the frequency of the two possible go S2s in the upcoming blocks. After the first four blocks, participants were told that the instructions would change in the remaining blocks, and therefore to contact the experimenter. Before the remaining blocks were started, the experimenter made sure that participants understood the altered instructions concerning the new frequency of the two go S2s. In general, the instructions emphasized speed and accuracy, and participants were asked to give R1 and R2 successively in fixed order, and to wait until the trial ended in case of a no-go trial. The stimulus-response mapping of all tasks, as well as the order of blocks (neutral vs. biased blocks first), and the S2 that occurred in $90 \%$ of go trials in biased blocks were counterbalanced across participants.

\section{Design and analysis}

Trials in which a response in Task 2 was required (i.e., a right index or middle finger response) were considered go trials, whereas trials in which a response in Task 2 was to be withheld were considered no-go trials. Data from the practice block were excluded from analyses. For the analysis of RTs, trials deviating more than 2.5 standard deviations from the individual cell means were considered outliers and excluded from analyses. Error rates in both tasks (ER1 and ER2) were arcsine transformed for statistical analyses, but raw ERs are reported as descriptive statistics. For Task 1, mean correct RT1s and ER1 were submitted to $2 \times 2$ analyses of variance (ANOVA), with trial type (go vs. no-go) and block type (neutral vs. biased) as repeated measures. For Task 2, mean correct RT2s were submitted to an ANOVA, with R2 frequency ${ }^{1}$

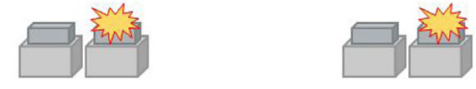

(neutral vs. frequent vs. less frequent) as a repeated measure. For ER2, an ANOVA with R2 frequency as a repeated measure for errors in go trials (a response was withheld when it was actually required) and an ANOVA with block type for errors in no-go trials (a response was given when it should be withheld) were calculated separately.

\section{Results}

Mean RT1s are shown in Fig. 2 (see also Table 1 for mean RT1s and ER1s), and all mean RT2s and ER2s are summarized in Table 2. For RT1, 2.73\% of all trials were considered outliers, and for RT2, $1.75 \%$ of all trials were considered outliers.

\section{Task 1}

The ANOVA yielded no significant main effect of trial type, $F(1,47)=0.48, p=.492, \eta_{\mathrm{p}}^{2}=.01$. The main effect of block type was significant, with RT1s being on average $70 \mathrm{~ms}$ longer in neutral $(712 \mathrm{~ms})$ relative to biased blocks $(642 \mathrm{~ms}), F(1$, $47)=28.37, p<.001, \eta_{\mathrm{p}}^{2}=.38$. Most importantly, the interaction was significant, $F(1,47)=69.57, p<.001, \eta_{\mathrm{p}}^{2}=.60$. The no-go BCE was present in biased blocks ( $63 \mathrm{~ms}), t(47)=5.62$, $p<.001, d=0.81$, and in neutral blocks, it was inverted $(-50$ $\mathrm{ms}), t(47)=-4.18, p<.001, d=-0.60$. Error rates were very low, but the analysis for ER1 revealed a significant main effect of trial type, with on average $1.30 \%$ more errors for go $(2.54 \%)$ relative to no-go trials $(1.24 \%), F(1,47)=53.25, p$

\footnotetext{
${ }^{1}$ This factor refers to the actual experimental manipulation. Note though that the critical assumption is that a frequent Task 2 response is prepared.
} 


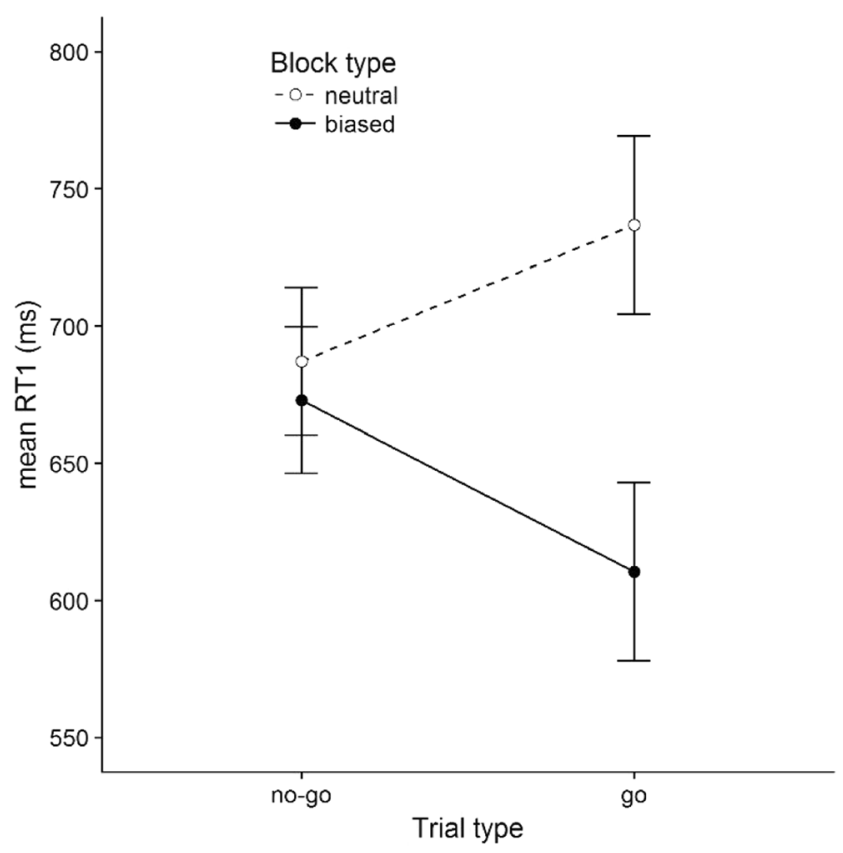

Fig. 2. Mean RTs of Task 1 (RT1) as a function of trial type (no-go vs. go) and block type (neutral vs. biased). Error bars are 95\% within-subject confidence intervals for the difference between block type (see Pfister \& Janczyk, 2013)

$<.001, \eta_{\mathrm{p}}^{2}=.53$. Neither the main effect of block type, $F(1,47)$ $=0.01, p=.915, \eta_{\mathrm{p}}^{2}<.01$, nor the interaction were significant, $F(1,47)=0.07, p=.798, \eta_{\mathrm{p}}^{2}<.01 .^{2}$

\section{Task 2}

The analysis for RT2 revealed a significant main effect of R2 frequency, $F(2,94)=64.75, p<.001, \eta_{\mathrm{p}}^{2}=.58$. On average the longest RT2s were observed for less frequent responses $(1,105$ $\mathrm{ms}$ ), with intermediate RT2s for neutral blocks (982 ms), and shortest RT2s for frequent responses $(783 \mathrm{~ms})$. All pairwise comparisons were significant (all $t \mathrm{~s} \geq 12.47$, all $p \mathrm{~s} \leq .001$ ). This suggests that participants prepared the more likely R2 alternative in advance. The ANOVA for ER2 in go trials yielded a significant main effect of $\mathrm{R} 2$ frequency, $F(2,94)=$ $21.25, p<.001, \eta_{\mathrm{p}}^{2}=.31$. On average ER2 for go trials in neutral blocks $(4.64 \%)$ was larger relative to frequent responses $(1.68 \%), t(47)=-7.83, p<.001, d=-1.13$, and relative to less frequent responses $(3.05 \%), t(47)=-5.29, p$ $<.001, d=-0.76$. However, ER2 neither differed between frequent and less frequent responses in go trials, $t(47)=$

\footnotetext{
${ }^{2}$ Note that the higher ER1 for go relative to no-go trials in biased blocks opposes the results pattern for RT1s, which suggests that a speed-accuracy trade-off (SAT; see, Liesefeld \& Janczyk, 2018) was present. This could potentially complicate the interpretation of the present results. An analysis of the potential influence of the SAT can be found in the Appendix. The critical interaction observed in RT1 was not compromised by an SAT.
}

Table 1 Mean response times (RT1, in ms) and error rates (ER1, in \%) for Task 1 as a function of trial type and block type

\begin{tabular}{|c|c|c|c|}
\hline & \multirow[b]{2}{*}{ Block type } & \multicolumn{2}{|c|}{ Trial type } \\
\hline & & No go & $\mathrm{Go}^{\mathrm{a}}$ \\
\hline \multirow[t]{2}{*}{ RT1 } & Neutral & 687 & 737 \\
\hline & Biased & 673 & 610 \\
\hline \multirow[t]{2}{*}{ ER1 } & Neutral & 1.30 & 2.49 \\
\hline & Biased & 1.18 & 2.60 \\
\hline
\end{tabular}

${ }^{\mathrm{a}}$ Mean RT1 and ER1 for biased blocks were also calculated separately for frequent and less frequent go responses in Task 2. RT1s for frequent responses were $587 \mathrm{~ms}$ and mean RT1s for less frequent responses were $856 \mathrm{~ms}$. ER1 for frequent responses was $2.39 \%$ and ER1 for less frequent responses was $4.51 \%$.

$0.24, p=.810, d=0.03$, nor between no-go trials of neutral and biased blocks, $F(1,47)=1.35, p=.251, \eta_{\mathrm{p}}^{2}=.03$.

\section{Discussion}

Usually, giving two temporally overlapping responses in two different tasks results in worse performance in one or both of the tasks - that is, most often prolonged RTs - in comparison with their isolated application. Such dual-task costs are a common observation, and only few exceptions were reported (see Janczyk, Pfister, Wallmeier, \& Kunde, 2014); thus, a possible beneficial aspect of dual tasking is only rarely considered (but see, e.g., Reissland \& Manzey, 2016). Yet there are situations in which giving two temporally overlapping responses can actually be at least less detrimental compared with giving only one response. Such a counterintuitive effect has been observed when Task 2 requires a response (go trial) compared with when Task 2 demands no response (no-go trial; e.g., Durst \& Janczyk, 2018; Miller, 2006). Presumably, this so-called

Table 2 Mean response times (RT2, in ms) and error rates (ER2, in \%) for Task 2 as a function of trial type and block type (R2 frequency)

\begin{tabular}{llll}
\hline & & \multicolumn{2}{l}{ Trial type } \\
\cline { 3 - 4 } & Block type (R2 frequency) & No go & Go \\
\hline RT2 & Neutral & & 982 \\
& Biased (frequent) & & 783 \\
& Biased (less frequent) & & 1105 \\
ER2 & Neutral & 0.26 & 4.64 \\
& Biased (frequent) & 1.15 & 1.68 \\
& Biased (less frequent) & & 3.05 \\
\hline
\end{tabular}

Note. In no-go trials of biased blocks, no distinction between frequent and less frequent responses in Task 2 can be made. Thus, only one mean ER2 for no-go trials in biased blocks can be reported 
no-go BCE arises from the inhibition of an already prepared Task 2 response, spilling over to Task 1, and impeding motor execution in this task (but see Röttger \& Haider, 2017, for an alternative explanation). To directly test this preparation hypothesis, we compared performance in two different blocks, where Task 2 was always a choice/no-go task: In neutral blocks, preparation of a particular go response was not encouraged, but in biased blocks, one response was required more frequently than the other, thus encouraging advance preparation of this particular response.

\section{Task 1 performance in no-go trials suffers only when Task 2 response preparation is encouraged}

The present results are straightforward. First, RT2s for frequent responses were shorter than those for less frequent responses, supporting the idea that participants indeed prepared for the more likely response alternative. Second, in neutral blocks, RT1 was shorter when Task 2 was a no-go trial, replicating results reported by Janczyk and Huestegge (2017). Most important, this pattern was reversed in biased blocks, thus the no-go BCE reemerged when preparation of a Task 2 response was encouraged. This result supports the preparation hypothesis and is also in line with the assumption that the inhibition of an already prepared response is the mechanism that underlies the no-go BCE. In a recent mental-chronometry study, Durst and Janczyk (2018) obtained further evidence that the no-go $\mathrm{BCE}$ arises during motor execution (i.e., the locus of the no-go BCE) of Task 1 when it temporally overlaps with the central stage (i.e., the source of the no-go BCE) of Task 2 (for an illustration see, Durst \& Janczyk, 2018, Fig. 1d). They suggested (motor) inhibition as the controlled process occurring in the central stage of Task 2 processing, causing the no-go BCE at the level of motor execution of Task 1. Note that the present results are not in line with an alternative explanation for the no-go BCE that was advanced by Röttger and Haider (2017). According to this account, a no-go trial automatically implies the activation of, for example, a no-go tag or the absence of sensory feedback when not responding - features that are on a more abstract level incompatible with Task 1 features. Thus, if this explanation were true, the same no-go BCE would have been expected regardless of block type.

\section{Possible objections and limitations}

Although the main results are straightforward, some aspects are worth discussing. One observation that appears not to be in line with the assumed inhibition in biased blocks is that RT1s for no-go trials are similar in neutral and biased blocks. At first glance, one would have expected longer no-go RT1s in biased compared with neutral blocks instead. We concur, but we believe that several explanations for this are conceivable.
Because of the block-wise application, we cannot exclude further differences that either decreased RT1s in biased blocks or increased RT1s in neutral blocks (or both), thereby obscuring the expected difference for no-go trials. For example, the possibility of preparation in biased blocks may have led to a better preparation in general, which decreased RT1s in biased blocks. Further, as RT2s were also longer for neutral than for biased blocks, even a small portion of grouped responses in neutral blocks may have increased RT1s accordingly.

A second objection concerns the nature of the neutral blocks in our experiment. One important advantage of our design is that - except for the distribution of the go-response frequencies-both blocks are comparable. However, one might suggest that in neutral blocks no inhibition occurred in no-go trials, because the two-choice/no-go task was more similar to a three-choice task. In this case, left versus right response code overlap may have induced a compatibilitybased BCE in go trials that may have prolonged RT1 in neutral blocks. It is, however, unclear whether such code overlap necessarily leads to interference. Rather, in case of two compatible responses, facilitation is likely as well. Further, an exploratory post hoc analysis revealed that such a compatibility-based BCE was not evident in the data, $t(47)=$ $-1.40, p=.168$, and numerically even inversed.

\section{Relations to other phenomena}

The present results can also be related to other phenomena. For example, a reduction in dual-task costs in Task 1 was also reported when saccadic eye movements were required in Task 2 as compared with keeping the eye fixated at the screen center (Huestegge \& Koch, 2014; see also Raettig \& Huestegge, 2018). These studies assumed that a saccadic eye movement toward a peripheral target occurs rather automatically, and thus inhibition is needed to keep the eye fixated at the screen center. Again, then, performing only one motor response is harder than performing two responses in this setup as well, and inhibition appears to be the underlying mechanism for this.

Our manipulation of stimulus and response frequency conceivably renders the less frequent stimuli unexpected. Unexpected events, in turn, are also known for slowing ongoing responses (Wessel, 2018; Wessel \& Aron, 2013), and this might have affected RT1s as well. In fact, in a post hoc exploration, RT1s were slower for less frequent Task 2 responses than for the frequent Task 2 responses. However, given that RT2s were slower as well, it is difficult to separate effects of unexpectedness from other possible sources with the present design. For example, RT1s may as well have been prolonged via response grouping (see Ulrich \& Miller, 2008) or because less frequent responses impose larger dual-task costs in general. Future research should try to separate unexpectedness 
from other sources to identify a possible unique contribution.

The present results further support the assumption that the no-go BCE is caused by inhibition of a prepared Task 2 response. As such it seems important to clearly distinguish the no-go BCE from other types of BCEs, which have different causes. The most-well known other type are compatibilitybased BCEs, which are likely caused by automatic response feature activation occurring in Task 2 (Hommel, 1998; Huestegge, Pieczykolan, \& Janczyk, 2018; Janczyk, Pfister, Hommel, \& Kunde, 2014; Janczyk et al., 2018; Lien \& Proctor, 2000; Logan \& Delheimer, 2001; Logan \& Schulkind, 2000; Oriet, Tombu, \& Jolicoeur, 2005; Renas, Durst, \& Janczyk, 2018; Schubert, Fischer, \& Stelzel, 2008; Watter \& Logan, 2006; see Durst \& Janczyk, 2019, for a direct comparison). To allow for a more precise distinction of backward crosstalk phenomena, future studies should investigate to what extent the no-go BCE differs from still other types of BCEs, which seem to be based on motor processes in Task 1, Task 2, or even both tasks (Miller \& Alderton, 2006; Ruiz Fernández \& Ulrich, 2010).

\section{Conclusion}

Motivated by the different suggestions advanced in the previous literature (Durst \& Janczyk, 2018; Janczyk \& Huestegge, 2017; Miller, 2006; Röttger \& Haider, 2017), the present study provides a direct test that preparation of a Task 2 response and its inhibition in no-go trials is the reason for performance decrements in Task 1, when Task 2 is a no-go task-a phenomenon known as the no-go BCE. More generally, the present results support the view that reduced preparation for Task 2 can reduce performance decrements in Task 1. Therefore, performing two motor responses at the same time can not only produce the usual observation of dual-task costs but can also, under certain circumstances, reduce these costs.

Authors note This research was supported by Grants JA2307/6-1 (awarded to M.J.) and UL116/15-1 (awarded to R.U.) within the Priority Program 1772 "Human Performance Under Multiple Cognitive Task Requirements: From Basic Mechanisms to Optimized Task Scheduling" of the German Research Foundation (Deutsche Forschungsgemeinschaft; DFG). Work of M.D. and M.J. was further supported by the Institutional Strategy of the University of Tübingen (ZUK 63). Data of this experiment is available at the Open Science Framework (https://osf.io/), where it can be accessed via the following link: https://osf.io/zpgr2

We thank Hilde Haider and Scott Watter for helpful and constructive comments on a previous version of this manuscript.

\section{Appendix}

\section{Speed-accuracy trade-off}

The higher ER1 for go relative to no-go trials in biased blocks opposes the results pattern for RT1s, where RT1s were shorter in go relative to no-go trials. This suggests that a speedaccuracy trade-off (SAT) was present, which could complicate the interpretation of the present results (although the descriptive difference for ER1 was small in general).

To investigate whether the SAT modulated the pattern of results obtained for RT1, the method of Janczyk (2016) was adopted. To this end, no-go BCEs for RT1 and ER1 were calculated for each participant. If the signs of both no-go BCEs did not match, an SAT for the respective participant was indicated. RT1 data were then submitted to a mixed ANOVA, with trial type and block type as repeated measures plus the additional between-subjects factor SAT.

The main effect of SAT was not significant, $F(1,46)=1.25$, $p=.270, \eta_{\mathrm{p}}^{2}=.03$. However, the interaction of trial type and SAT was significant, $F(1,46)=6.38, p=.015, \eta_{\mathrm{p}}^{2}=.12$. The interaction of block type and SAT was not significant, $F(1,46)$ $=0.47, \mathrm{p}=.498, \eta_{\mathrm{p}}^{2}=.01$. Most crucially, the interaction of trial type and block type was significant, $F(1,46)=64.20, p<$ $.001, \eta_{\mathrm{p}}^{2}=.58$, but the interaction of SAT, trial type, and block type was not significant, $F(1,46)=0.01, p=.931, \eta_{\mathrm{p}}^{2}<.01$. Additional separate analyses for participants with and without an SAT yielded qualitatively similar results.

Taken together, the results of the SAT analysis indicate that the SAT did not modulate the crucial interaction of trial type and block type, as the three-way interaction was not significant. This renders the interpretation of the present results less problematic.

\section{References}

Cohen, J. (1988). Statistical power analysis for the behavioral sciences (2nd ed.). Hillsdale, NJ: Erlbaum.

Donders, F. C. (1969). On the speed of mental processes. Acta Psychologica, 30, 412-431.

Durst, M., \& Janczyk, M. (2018). The motor locus of no-go backward crosstalk. Journal of Experimental Psychology: Learning, Memory, and Cognition, 44, 1931-1946.

Durst, M., \& Janczyk, M. (2019). Two types of backward crosstalk: Sequential modulations and evidence from the diffusion model. Acta Psychologica, 193, 132-152.

Hommel, B. (1998). Automatic stimulus-response translation in dualtask performance. Journal of Experimental Psychology: Human Perception and Performance, 24, 1368-1384.

Huestegge, L., \& Koch, I. (2014). When two actions are easier than one: How inhibitory control demands affect response processing. Acta Psychologica, 151, 230-236. 
Huestegge, L., Pieczykolan, A., \& Janczyk, M. (2018). Backward crosstalk and the role of dimensional overlap within and between tasks. Acta Psychologica, 188, 139-147.

Janczyk, M. (2016). Sequential modulation of backward crosstalk and task-shielding in dual-tasking. Journal of Experimental Psychology: Human Perception and Performance, 42, 631-647.

Janczyk, M., \& Huestegge, L. (2017). Effects of a no-go Task 2 on Task 1 performance in dual- tasking: From benefits to costs. Attention, Perception, \& Psychophysics, 79, 796-806.

Janczyk, M., Pfister, R., Hommel, B., \& Kunde, W. (2014). Who is talking in backward crosstalk? Disentangling response-from goalconflict in dual-task performance. Cognition, 132, 30-43.

Janczyk, M., Pfister, R., Wallmeier, G., \& Kunde, W. (2014). Exceptions to the PRP effect? A comparison of prepared and unconditioned reflexes. Journal of Experimental Psychology: Learning, Memory, and Cognition, 40, 776-786.

Janczyk, M., Renas, S., \& Durst, M. (2018). Identifying the locus of compatibility-based backward crosstalk: Evidence from an extended PRP paradigm. Journal of Experimental Psychology: Human Perception and Performance, 44, 261-276.

Ko, Y.-T., \& Miller, J. (2014). Locus of backward crosstalk effects on Task 1 in a psychological refractory period task. Experimental Psychology, 61, 30-37.

Kühn, S., Elsner, B., Prinz, W., \& Brass, M. (2009). Busy doing nothing: Evidence for nonaction-effect binding. Psychonomic Bulletin \& Review, 16, 542-549.

Lien, M.-C., \& Proctor, R. W. (2000). Multiple spatial correspondence effects on dual-task performance. Journal of Experimental Psychology: Human Perception and Performance, 26, 1260-1280.

Liesefeld, H. R., \& Janczyk, M. (2018). Combining speed and accuracy to control for speed-accuracy trade-offs(?). Behavior Research Methods, 1-21. https://doi.org/10.3758/s13428-018-1076-x

Logan, G. D., \& Delheimer, J. A. (2001). Parallel memory retrieval in dual-task situations: II. Episodic memory. Journal of Experimental Psychology: Learning, Memory, and Cognition, 27, 668-685.

Logan, G. D., \& Schulkind, M. D. (2000). Parallel memory retrieval in dual-task situations: I. Semantic memory. Journal of Experimental Psychology: Human Perception and Performance, 26, 1072-1090.

Miller, J. (2006). Backward crosstalk effects in psychological refractory period paradigms: Effects of second-task response types on first-task response latencies. Psychological Research, 70, 484-493.

Miller, J., \& Alderton, M. (2006). Backward response-level crosstalk in the psychological refractory period paradigm. Journal of Experimental Psychology: Human Perception and Performance, $32,149-165$.

Miller, J., \& Durst, M. (2014). "Just do it when you get a chance": the effects of a background task on primary task performance. Attention, Perception, \& Psychophysics, 76, 2560-2574.
Miller, J., \& Durst, M. (2015). A comparison of the psychological refractory period and prioritized processing paradigms: Can the responseselection bottleneck model explain them both? Journal of Experimental Psychology: Human Perception and Performance, 41, 1420-1441.

Oriet, C., Tombu, M., \& Jolicoeur, P. (2005). Symbolic distance affects two processing loci in the number comparison task. Memory \& Cognition, 33, 913-926.

Pfister, R., \& Janczyk, M. (2013). Confidence intervals for two sample means: Calculation, interpretation, and a few simple rules. Advances in Cognitive Psychology, 9, 74-80.

Raettig, T., \& Huestegge, L. (2018). The hard work of doing nothing: Accounting for inhibitory costs during multiple action control. Attention, Perception, \& Psychophysics, 80, 1660-1666.

Reissland, J., \& Manzey, D. (2016). Serial or overlapping processing in multitasking as individual preference: Effects of stimulus preview on task switching and concurrent dual-task performance. Acta Psychologica, 168, 27-40.

Renas, S., Durst, M., \& Janczyk, M. (2018). Action effect features, but not anatomical features, determine the backward crosstalk effect: Evidence from crossed-hands experiments. Psychological Research, 82, 970-980.

Röttger, E., \& Haider, H. (2017). Investigating the characteristics of "not responding": Backward crosstalk in the PRP paradigm with forced vs. free no-go decisions. Psychological Research, 81, 596-610.

Ruiz Fernández, S., \& Ulrich, R. (2010). Late backward effects in the refractory period paradigm: Effects of Task 2 execution on Task 1 performance. Psychological Research, 74, 378-387.

Schubert, T., Fischer, R., \& Stelzel, C. (2008). Response activation in overlapping tasks and the response-selection bottleneck. Journal of Experimental Psychology: Human Perception and Performance, 34, 376-397.

Ulrich, R., \& Miller, J. (2008). Response grouping in the psychological refractory period (PRP) paradigm: Models and contamination effects. Cognitive Psychology, 57, 75-121.

Watter, S., \& Logan, G. D. (2006). Parallel response selection in dual-task situations. Perception \& Psychophysics, 68, 254-277.

Wessel, J. R. (2018). Surprise: A more realistic framework for studying action stopping? Trends in Cognitive Sciences, 22, 741-744.

Wessel, J. R., \& Aron, A. R. (2013). Unexpected events induce motor slowing via a brain mechanism for action-stopping with global suppressive effects. The Journal of Neuroscience, 33, 18481-18491.

Publisher's note Springer Nature remains neutral with regard to jurisdictional claims in published maps and institutional affiliations. 